\title{
Stimulation of the $\alpha 7$ Nicotinic Acetylcholine Receptor Protects against Neuroinflammation after Tibia Fracture and Endotoxemia in Mice
}

\author{
Niccolò Terrando, ${ }^{1,2}$ Ting Yang, ${ }^{1,2}$ Jae Kyu Ryu, ${ }^{3}$ Phillip T Newton, ${ }^{1,4}$ Claudia Monaco, ${ }^{5}$ Marc Feldmann, ${ }^{5}$ \\ Daqing $\mathrm{Ma}^{6}{ }^{6}$ Katerina Akassoglou, ${ }^{3,7}$ and Meroyn Maze ${ }^{2}$
}

${ }^{1}$ Department of Physiology and Pharmacology, Karolinska Institute, Stockholm, Sweden; ${ }^{2}$ Department of Anesthesia and Perioperative Care, UCSF Medical Center, San Francisco, California, United States of America; ${ }^{3}$ Gladstone Institute of Neurological Disease, University of California San Francisco, San Francisco, California, United States of America; ${ }^{4}$ Department of Women's and Children's Health, Astrid Lindgren Children's Hospital, Karolinska University Hospital, Stockholm, Sweden; ${ }^{5}$ Kennedy Institute of Rheumatology, Nuffield Department of Orthopaedics, Rheumatology and Musculoskeletal Sciences, University of Oxford, London, United Kingdom; ${ }^{6}$ Anaesthetics, Pain Medicine and Intensive Care, Department of Surgery and Cancer, Faculty of Medicine, Imperial College London, Chelsea \& Westminster Hospital, London, United Kingdom; and ${ }^{7}$ Department of Neurology, University of California San Francisco, San Francisco, California, United States of America

\begin{abstract}
Surgery and critical illness often associate with cognitive decline. Surgical trauma or infection can lead independently to learning and memory impairments via similar, but not identical, cellular signaling of the innate immune system that promotes neuroinflammation. In this study we explored the putative synergism between aseptic orthopedic surgery and infection, the latter reproduced by postoperative lipopolysaccharide (LPS) administration. We observed that surgery and LPS augmented systemic inflammation up to postoperative d 3 and this was associated with further neuroinflammation (CD1 1 b and CD68 immunoreactivity) in the hippocampus in mice compared with those receiving surgery or LPS alone. Administration of a selective $\alpha 7$ subtype nicotinic acetylcholine receptor ( $\alpha 7 \mathrm{nAChR}$ ) agonist $2 \mathrm{~h}$ after LPS significantly improved neuroinflammation and hippocampaldependent memory dysfunction. Modulation of nuclear factor-kappa B (NF- $\mathrm{B}$ ) activation in monocytes and regulation of the oxidative stress response through nicotinamide adenine dinucleotide phosphate (NADPH) signaling appear to be key targets in modulating this response. Overall, these results suggest that it may be conceivable to limit and possibly prevent postoperative complications, including cognitive decline and/or infections, through stimulation of the cholinergic antiinflammatory pathway.
\end{abstract}

Online address: http://www.molmed.org

doi: $10.2119 / \mathrm{molmed} .2014 .00143$

\section{INTRODUCTION}

Acute illness and hospitalization is often accompanied by learning and memory impairments, especially among elderly patients, a steadily growing portion of the surgical and intensive care unit (ICU) populations (1). Postoperative cognitive dysfunction (POCD) associates with higher morbidity and mortality (even when propensity matched for comorbidities), including increase in functional disability and rates of admission to long-term care institutions; the presence of postoperative cognitive decline significantly increases the costs to healthcare (2). Although the mechanisms underly-

Address correspondence to Niccolò Terrando, Karolinska Institutet, Department of Physiology and Pharmacology, Nanna Svart väg 2, Stockholm, 171 77, Sweden. Phone: +46-8-524872-41; Fax: +46-8-524-872-66; E-mail: niccolo.terrando@ki.se.

Submitted July 21, 2014; Accepted for publication October 29, 2014; Epub (www.molmed.org) ahead of print October 29, 2014.

\section{The Feinstein Institute for Medical Research Empowering Imagination. Pioneering Discovery.



ing changes in acute mental function remain unclear, multiple risk factors, in particular age, surgical procedures (including cardiac and orthopedic in particular) and infections, have been linked to the development of POCD $(3,4)$. Neuroinflammation initiated by extra-central nervous system (CNS) surgical trauma, has been advanced as a key component in the pathogenesis of surgery-induced cognitive dysfunction; however, the mechanisms whereby neuroinflammation impairs behavior in the perioperative period remain unclear (5).

There is increasing evidence that the CNS can regulate immune and inflammatory processes (6). Soluble mediators from the periphery can exert multiple effects on the brain, contributing to "sick- 
ness behavior" and memory impairments (7). Preclinical models suggest a key role for the innate immune response, including release of systemic cytokines such as tumor necrosis factor $\alpha$ (TNF- $\alpha)$, interleukin (IL)-1 $\beta$ and high mobility group box 1 (HMGB-1) in contributing to neuroinflammation and cognitive decline (8-10). Direct neural pathways including cholinergic signaling via the vagus nerve and the $\alpha 7$ nicotinic acetylcholine receptor $(\alpha 7 \mathrm{nAChR})$ regulate the acute and chronic inflammatory response (11). In surgery-induced inflammation, we reported that $\alpha 7 \mathrm{nAChR}$ signaling attenuates postoperative cognitive decline by modulating endothelia function at the blood-brain barrier (BBB) and preventing macrophage infiltration into the CNS (12).

In the present study, we sought to explore the effect of lipopolysaccharide (LPS), a surrogate for postoperative infection, on surgery-induced neuroinflammation and cognitive decline. Since postoperative complications, in particular infective and respiratory complications, have been significantly associated with prolonged postoperative cognitive decline (3), we combined our surgical model with endotoxemia to test the effects of a selective $\alpha 7 \mathrm{nAChR}$ agonist on cognitive function and neuroinflammation in this two-hit model. Herein, we demonstrate that postoperative LPS exposure prolongs the inflammatory response induced by the surgical procedure and that activation of endogenous inflammatory-resolving mechanisms via stimulation of the $\alpha 7 \mathrm{nAChR}$ signaling pathway attenuates the cognitive dysfunction.

\section{MATERIALS AND METHODS}

\section{Animals and Surgery}

Wild-type male C57BL/6J mice (12 wks old) were obtained from the Jackson Laboratory (Bar Harbor, ME, USA). All animals were fed standard rodent chow and water ad libitum, and housed ( $<$ five mice/cage) in sawdust-lined cages in an air-conditioned environment with $12 \mathrm{~h}$ light-dark cycles. Seven days of acclimatization were allowed before starting any experiment. All animals were monitored on a daily basis for general body conditions. Experiments were conducted under the UK Home Office and IACUC, University of California (San Francisco, CA, USA) approved licenses.

Animals were anaesthetized with isoflurane at a minimum alveolar concentration (MAC) of $1.5 \pm 0.2$ (corresponding to an end-tidal concentration of $2.1 \%$ isoflurane) at $30 \% \mathrm{FiO}_{2}$ and combined with buprenorphine (Buprenex, $0.1 \mathrm{mg} / \mathrm{kg}$ subcutaneously [s.c.]). An open tibial fracture was performed under aseptic conditions as described previously (13). A middle incision was performed on the left hind paw and a $0.38-\mathrm{mm}$ pin was inserted into the intramedullary canal, the periosteum stripped and osteotomy performed. Drugs were diluted in $0.9 \%$ saline prior to use and injected as indicated. Mice were then allowed to recover from surgery and anesthesia and were subjected to an intraperitoneal (IP) injection of either vehicle (S-group) or LPS (S+LPSgroup) derived from Escherichia coli endotoxin (0111:B4, $1 \mathrm{mg} / \mathrm{kg}$ ) (InvivoGen, San Diego, CA, USA) at $24 \mathrm{~h}$ postoperatively. The $\alpha 7 \mathrm{nAChR}$ agonist PHA 568487 (0.4 mg/kg) (12) (Tocris Bioscience, Ellisville, MO, USA) (S+LPS+PHA-group) was administered IP $2 \mathrm{~h}$ following LPS administration when sickness became noticeable in behavior.

\section{Cytokine Measurement}

Blood was sampled transcardially after thoracotomy under terminal anesthesia 3 and $7 \mathrm{~d}$ in separate cohorts and centrifuged at 2,000 $\mathrm{g}$ for $7 \mathrm{~min}$ at $4^{\circ} \mathrm{C}$. Blood samples taken from animals without any interventions served as controls. Plasma samples were stored at $-20^{\circ} \mathrm{C}$ for further analysis. Plasma cytokines were measured by enzyme-linked immunosorbent assay (ELISA) according to the manufacturer's instruction (Biosource, Camarillo, CA, USA). The sensitivities of the assays were $<7 \mathrm{pg} / \mathrm{mL}$ for IL- $1 \beta$, and $<3 \mathrm{pg} / \mathrm{mL}$ for IL-6.

\section{Immunohistochemistry (IHC)}

Mice were deeply anesthetized with isoflurane and perfused transcardially with ice-cold heparinized $0.1 \mathrm{~mol} / \mathrm{L}$ phosphate buffer solution (PBS) followed by $4 \%$ paraformaldehyde (PFA) in $0.1 \mathrm{~mol} / \mathrm{L}$ PBS at pH 7.4 (VWR International, East Grinstead, UK). The brains were harvested and postfixed in $4 \%$ PFA at $4^{\circ} \mathrm{C}$ overnight and cryoprotected in 15\% sucrose for $24 \mathrm{~h}$ (VWR International) and then $30 \%$ sucrose for a further $48 \mathrm{~h}$. Brain tissue was then embedded in OCT (VWR International), coronal sections were cut at $25 \mu \mathrm{m}$ and mounted on Superfrost plus slides (Menzel-Glaser, Braunschweig, Germany). The sections were quenched for $30 \mathrm{~min}$ with $0.3 \%$ hydrogen peroxide in $70 \%$ methanol and blocked in 3\% normal goat serum for $1 \mathrm{~h}$, then incubated in rat anti-CD11b (1:200, low endotoxin, clone M1/70.15, Serotec, Oxford, UK) overnight at $4^{\circ} \mathrm{C}$. A goat anti-rat secondary antibody (1:200, Chemicon International, CA, USA) was applied for $1 \mathrm{~h}$, the staining was revealed by the $\mathrm{ABC}$ reagent (standard Vectastain ABC Elite Kit, Vector Labs, Cambridge, UK) and 3,3'diaminobenzidine (DAB) (Vector Labs). To label monocytes/macrophages, sections were incubated in a rabbit antiCD68 (1:250, Abcam, Cambridge, UK) for $24 \mathrm{~h}$ at $4^{\circ} \mathrm{C}$ followed by a goat anti-rabbit Cy3 conjugated secondary antibody (1:500, Chemicon International, Temecula, CA, USA) for $1 \mathrm{~h}$ at room temperature in dark. A negative control omitting the primary antibody was performed in all experiments. Photomicrographs were acquired with a digital microscope (Axiocam, Carl Zeiss, Thornwood, NY, USA) and immunoreactivity was calculated using a $0.4 \mathrm{~mm}^{2}$ frame in three coronal hippocampal regions. Image quantification was analyzed with ImageJ software (NIH, Bethesda, MD, USA; http://imagej.nih.gov/ij/) as described previously (12).

\section{Bone Marrow-Derived Macrophages (BMDMs)}

Briefly, bone marrow (BM) was isolated from the tibia and femur of $10-w k-$ 
old mice. BM cells were cultured in RPMI 1640 (Invitrogen [Thermo Fisher Scientific Inc., Waltham, MA, USA]) supplemented with $10 \%$ (vol/vol) heat-inactivated fetal bovine serum (Invitrogen [Thermo Fisher Scientific]), 1\% penicillin-streptomycin (Invitrogen [Thermo Fisher Scientific]), and $10 \mathrm{ng} / \mathrm{mL}$ murine M-CSF (eBioscience, San Diego, CA, USA). On d 6, adherent BMDMs were harvested from plates by the addition of PBS containing $5 \mathrm{mmol} / \mathrm{L}$ ethylenediaminetetracetic acid for experiments. BMDMs were plated and cultured with 1\% fetal bovine serum in RPMI 1640 overnight in Nunc plates. Cells were preincubated with PHA 568487 (10 $\mu \mathrm{g} / \mathrm{mL})$ for $30 \mathrm{~min}$ followed by mouse LPS (100 ng/mL) stimulation for $2 \mathrm{~h}$. The cells were then fixed with $4 \%$ paraformaldehyde for $15 \mathrm{~min}$ at room temperature, rinsed in PBS and incubated with blocking buffer (PBS containing 5\% normal goat serum and $0.3 \%$ Triton X-100) for $60 \mathrm{~min}$. Cells were stained with anti-phospho-NF-кB p65 (1:200) (Cell Signaling Technology, Danvers, MA, USA) for $24 \mathrm{~h}$ at $4^{\circ} \mathrm{C}$. Cells were washed in PBS and incubated with anti-rabbit Cy3-conjugated secondary antibody (1:200) (Jackson ImmunoResearch, West Grove, PA, USA) for $1.5 \mathrm{~h}$ at room temperature in the dark. After washing with PBS, coverslips were mounted using ProLong Gold antifade reagent (Invitrogen [Thermo Fisher Scientific]). Five representative images per well were taken using an Axioplan II epifluorescence microscope (Carl Zeiss) with a dry Plan-Neofluar 400.75 NA objective and an AxiocamHRc CCD camera. Images were acquired and analyzed with AxioVision image analysis software. Quantification was determined as the proportion of total cells exhibiting nuclear phospho-NF-кB p65. All experiments were repeated three times.

Another batch of BMDMs were stimulated with LPS for $24 \mathrm{~h}$. Cells were then incubated in DPBS for $10 \mathrm{~min}$ at $37^{\circ} \mathrm{C}$ and pipetted gently multiple times to obtain the cell suspension. Nicotinamide adenine dinucleotide phosphate-
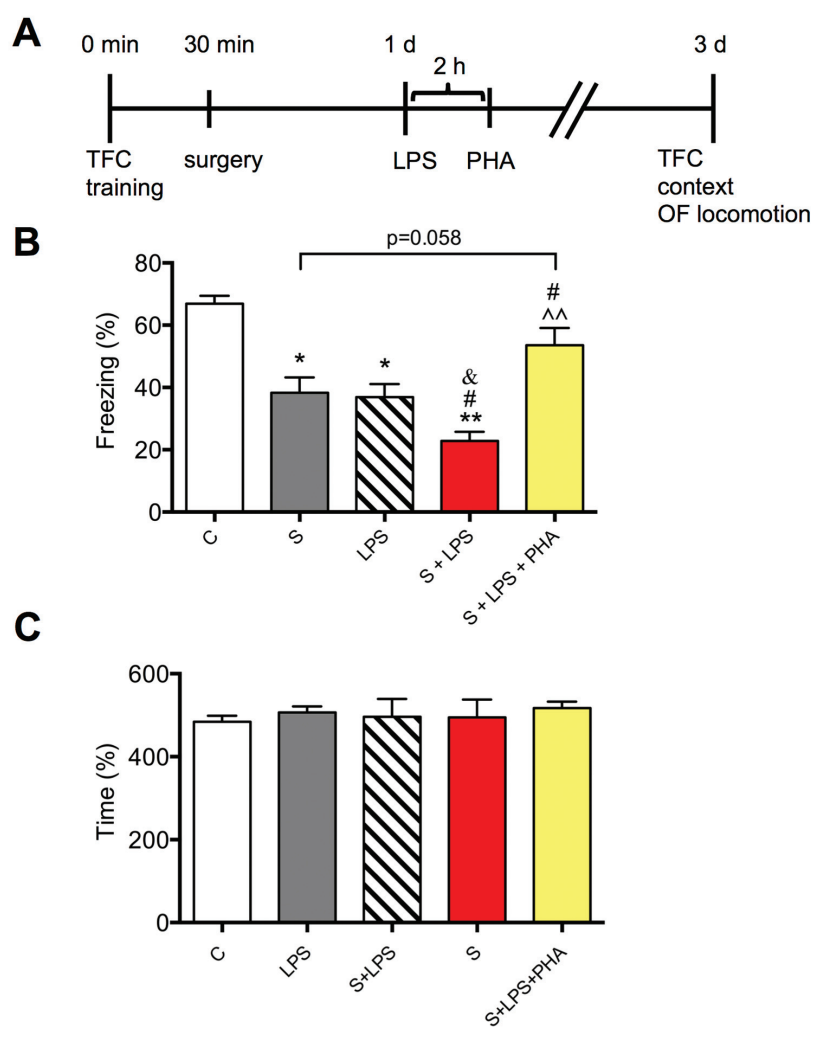

Figure 1. Cholinergic stimulation improves cognitive outcome after surgery and LPS administration. (A) Mice were operated on within 30 min after training. At $24 \mathrm{~h}$ the S+LPSgroup received $1 \mathrm{mg} / \mathrm{kg}$ LPS and a bolus injection of PHA 568487 or saline after $2 \mathrm{~h}$. (B) Contextual fear response, as measured by freezing behavior, is impaired in animals receiving surgery followed by LPS exposure as compared with nailve (saline injected) or surgery groups. Administration of a selective $\alpha 7 \mathrm{nAChR}$ agonist (PHA) significantly improved the cognitive abnormality. (C) Open field was performed after TFC to measure general locomotor activity for a duration of 5 min; no differences in general activity were observed among groups. Data are expressed as mean $\pm \mathrm{SEM}, \mathrm{n}=10$. ( ${ }^{*} P<0.05$ versus $\mathrm{C}$; ${ }^{* *} P<0.01$ versus $C$; ${ }^{\#} P<0.05$ versus $S$; ${ }^{\circledR} P<0.05$ versus LPS; ${ }^{\wedge} P<0.01$ versus $S+L P S$ ). C, control; $S$, surgery; PHA, PHA 568487; TFC, trace fear conditioning; OF, open field.

mediated (NADPH-mediated) superoxide generation was detected by AutoLumat LB953 Multi-Tube Luminometer (Berthold Technologies, Bad Wildbad, Germany) as described previously (14). NADPH (100 $\mu \mathrm{mol} / \mathrm{L}$, Sigma-Aldrich) and lucigenin (5 $\mu \mathrm{mol} / \mathrm{L}$, Sigma-Aldrich) were added to the cell suspension, superoxide level was determined by measuring lucigenin chemiluminescence every $3 \mathrm{~s}$ for $3 \mathrm{~min}$.

\section{In Vitro Chondrogenic Differentiation Model}

The nontransformed mesenchymal rat chondrogenic cell-line RCJ3.1C5.18
(C5.18) was obtained from Anna Spagnoli (15). C5.18 cells were maintained in minimal essential medium containing 15\% FBS with $10^{-7} \mathrm{~mol} / \mathrm{L}$ dexamethasone (16). Cells were seeded in maintenance medium, and, once confluent, subsequently supplemented with $50 \mu \mathrm{g} / \mathrm{mL}$ ascorbic acid (Sigma-Aldrich, St. Louis, MO, USA; A5960) and $1 \mathrm{mmol} / \mathrm{L} \beta$-glycerophosphate (BGP) (Sigma-Aldrich, G9422) to stimulate chondrogenic differentiation, in the presence of $10 \mu \mathrm{g} / \mathrm{mL}$ PHA 568487 or PBS vehicle. Alcian blue staining and quantification was performed as described previously (17). Quantification of colonies was performed using ImageJ 



Figure 2. PHA 568487 effects on CD1 1 b activation following surgery and LPS. Hippocampi were harvested at 3 and $7 \mathrm{~d}$ after surgery/LPS administration and stained with anti-CD $11 \mathrm{~b}$. Photomicrographs show CA1 of control animals (C), surgery (S), lipopolysaccharide (LPS), surgery with postoperative LPS (S+LPS) and in combination with a bolus dose of a selective $\alpha 7$ nAChR agonist (S+LPS+PHA) at $3 \mathrm{~d}$. Higher CD1 1 b immunoreactivity was observed after surgery and LPS exposure at both 3 and $7 \mathrm{~d}(P<0.01, P<0.05$ respectively). Significant hypertrophy of the cell bodies and loss of pseudopodia occurred in LPS-treated mice after orthopedic surgery; this was attenuated by administration of PHA $5684872 \mathrm{~h}$ after LPS onset $(P<0.01$ versus $S+L P S)$. Reactive microglia/monocytes were present up to $d 7$ in the S+LPS group and returned to baseline in the other treatment groups. Data are expressed as mean \pm SEM and compared by one-way ANOVA and Student-Newman-Keuls method, $n=4{ }^{* *} P<$ 0.01 versus $C$; ${ }^{~} P<0.05$ versus $\mathrm{S}$; ${ }^{\#} P<0.01$ versus $\mathrm{S}$; ${ }^{\& \&} P<0.05$ versus $L P S$; ${ }^{\wedge} P<0.05$ versus $S+L P S$; ${ }^{\wedge} P>0.01$ versus S+LPS). Scale bar, $50 \mu \mathrm{m}$. C, control; S, surgery; PHA, PHA 568487.

software (NIH) by setting color and size thresholds. For all treatment groups, $\mathrm{n}=$ 6 wells.

\section{Contextual Fear Conditioning}

Behavior was performed in a dedicated fear-conditioning chamber (Med Associates Inc., St Albans, VT, USA). Mice are trained to associate an environment (context) with a conditional stimulus (tone) and an unconditional stimulus (foot shock). Training paradigm was per- formed as described previously (8): tone duration $20 \mathrm{~s}$, level $75 \mathrm{~dB}$; shock duration $2 \mathrm{~s}$, and intensity $0.75 \mathrm{mAmp}$. Mice were trained before surgery and all mice received the same handling regardless of the final group allocation. During training, an initial exploratory phase (100 s) was followed by two trials separated by a 100-s intertrial interval. Trials consisted of a $20 \mathrm{~s}$ auditory cue $(75-80 \mathrm{~dB}, 5 \mathrm{k} \mathrm{Hz}$, conditional stimulus) followed by a 2-s foot shock (0.75 mAmp, unconditional stimu- lus). Contextual assessment was performed $72 \mathrm{~h}$ after surgery in the same chamber but with no cues (tone or shock). Freezing behavior, excluding breathing and movement of vibrissae, was automatically scored for $270 \mathrm{~s}$ by the video tracking software. Freezing scores for each subject were expressed as a percentage for each portion of the test. Memory for the context (contextual memory) for each subject was obtained by subtracting the percent freezing in the novel environment from that in the context.

General locomotion activity was measured after contextual assessment using an automated photobeam activity system (Aditech, Fjärås, Sweden) with a 5 min exploration period. All assessments were performed in a blinded fashion.

\section{Data Analysis}

Statistical analyses were performed using GraphPad Prism version 5.0d (GraphPad Software, San Diego, CA, USA). The results are expressed as mean \pm SEM. Data were analyzed with analysis of variance followed by Newman-Keuls post hoc test wherever appropriate. A $P<0.05$ was considered to be a statistical significance.

\section{RESULTS}

\section{Cholinergic Agonist Improves LPS- Exacerbated Cognitive Decline}

To understand the contribution of surgical trauma and infection on memory function, we administered LPS systemically $24 \mathrm{~h}$ after orthopedic surgery to recreate a clinical scenario of postoperative wound infection (Figure 1A). To assess hippocampal-dependent memory function, we trained mice $30 \mathrm{~min}$ before surgery using trace fear conditioning and performed contextual assessment on postoperative d $3(8,9)$. Mice exposed to surgery and postoperative LPS exhibited a significant reduction in cognitive function (freezing S+LPS-group $22.86 \pm 2.9$ versus $C$-group $66.88 \pm 2.5, P<0.01$ ) when compared with either surgical or LPS-exposed animals only (freezing S-group $38.25 \pm 4.9$ versus LPS-group 
$36.88 \pm 4.2$, Figure $1 \mathrm{~B}, P<0.05)$. To attempt to ameliorate this cognitive impairment caused by surgery and LPS, we injected intraperitoneally a selective $\alpha 7 \mathrm{nAChR}$ agonist (PHA 568487) $2 \mathrm{~h}$ following endotoxemia and tested the contextual memory on d 3. Treatment with PHA 568487 significantly improved the freezing behavior attenuating the memory dysfunction caused by the two-hit provocation (freezing S+LPS+PHA-group $53.57 \pm 5.5, P<0.01)$. To exclude locomotor impairments or general immobility due to sickness behavior, mice were tested in an open field and no significant changes were observed between groups (Figure 1C). Together, these results suggest a use for cholinergic agonists in limiting adverse cognitive outcome caused by trauma and endotoxemia.

\section{$\alpha 7$ nAChR Inhibits Hippocampal CD1 1b/CD68 Activation}

To understand how PHA 568487 protected from further memory decline, we assessed neuroinflammation in the hippocampus on postoperative $\mathrm{d} 3$ and 7 . We used CD11b to visualize activated microglia and recruited macrophages (antigenically indistinguishable) and CD68 to further highlight the process of active phagocytosis in the hippocampus (18). Densitometry of CD11b immunostaining revealed significantly higher immunoreactivity with hypertrophy of cell bodies and clumping of the ramifications, characteristic of activation, following the combination of surgery with LPS as compared with surgery or LPS only $(P<0.05$, Figure 2$)$. Both surgery and LPS activated immunocompetent cells in the CNS, including $\mathrm{CD}^{+} 8^{+}$cells, yet the combination of these challenges significantly enhanced stimulated microglia/ monocytes in the hippocampus after $3 \mathrm{~d}$ and this persisted for up to postoperative d $7(P<0.01$, Figure 3$)$.

Monocytes and microglia widely express $\alpha 7$ nAChR (19). Notably, treatment with PHA 568487 significantly reduced the activation of $\mathrm{CD}^{+} 8^{+}$and $\mathrm{CD} 11 \mathrm{~b}^{+}$cells in the hippocampus at $\mathrm{d} 3(P<0.01$ respectively) and prevented its upregula-


Figure 3. Cholinergic agonist reduces reactive macrophages/microglia in the hippocampus after surgery and LPS. Hippocampi were harvested at 3 and $7 \mathrm{~d}$ after surgery/LPS administration and stained with anti-CD68. Epifluorescence shows enhanced immunoreactivity $3 \mathrm{~d}$ after surgery (S) and lipopolysaccharide (LPS) exposure. Postoperative infection (S+LPS) further increased the number of $\mathrm{CD} 8^{+}$cells, persisting up to $d 7$. Administration of a single bolus dose of a selective $\alpha 7$ nAChR agonist (PHA 568487) significantly reduced levels of CD68 ${ }^{+}$immunoreactivity, returning to baseline at $7 \mathrm{~d}$. Nuclei are counterstained with DAPI. Data are expressed as mean \pm SEM and compared by one-way ANOVA and Student-Newman-Keuls method, $\mathrm{n}=4$ ( ${ }^{* *} P<0.01$ versus $C$; ${ }^{\#} P<0.05$ versus $\mathrm{S}$; ${ }^{\# \#} P<0.01$ versus $\mathrm{S}$; ${ }^{\& \&} P<0.01$ versus LPS; ${ }^{\wedge \wedge} P<0.01$ versus S+LPS). Scale bar, $30 \mu \mathrm{m}$. C, control, S, surgery; PHA, PHA 568487.

tion at $\mathrm{d} 7(P<0.01$ and $P<0.05$ respectively) in surgical mice following LPS administration, suggesting that $\alpha 7 \mathrm{nAChR}$ negatively regulates neuroinflammation and microglia/macrophage activation (Figures 2, 3).

\section{Systemic Antiinflammatory Effects after Postoperative LPS Administration}

Prior work implicated a role for the systemic inflammatory milieu in modulating neuroinflammation and cognitive function (20). Transient changes in proinflammatory cytokines are observed after surgery and appear to contribute to the pathogenesis of postoperative cognitive decline. Systemic levels of IL-1 $\beta$ and IL-6 were increased significantly up to $\mathrm{d} 3$ combining surgery and LPS exposure. Compared to surgery or LPS alone, the double hit (S+LPS) group had a significant increase in both IL-1 $\beta$ (43.13 \pm $4.5 \mathrm{pg} / \mathrm{mL}$, S+LPS versus $\mathrm{S}, P<0.01$, Figure 4A) and IL-6 $(283 \pm 31.16 \mathrm{pg} / \mathrm{mL}$,
S+LPS versus $S, P<0.01$, Figure 4B) after $3 \mathrm{~d}$, with levels returning to baseline by $\mathrm{d} 7$.

Administration of PHA 568487 significantly attenuated the proinflammatory response reducing levels of both IL-1 $\beta$ $(7.6 \pm 2.63 \mathrm{pg} / \mathrm{mL}, \mathrm{S}+\mathrm{LPS}$ versus S+LPS+PHA, $P<0.01$, Figure 4A) and IL-6 (109.1 $\pm 11.53 \mathrm{pg} / \mathrm{mL}$, S+LPS versus S+LPS+PHA, $P<0.05$, Figure 4B).

\section{Effects of $\alpha 7$ nAChR Stimulation on Bone Marrow-Derived Macrophages and Chondrocytes In Vitro}

Macrophage activity is required to mediate surgery-induced neuroinflammation $(12,21)$ and bone marrow-derived cells are integral to a functioning inflammatory reflex (22). To better understand the antiinflammatory effects of cholinergic stimulation, we incubated bone marrow-derived macrophages (BMDMs) with the selective $\alpha 7$ cholinergic receptor agonist (PHA 568487, $10 \mu \mathrm{g} / \mathrm{mL}$ ) and 
A

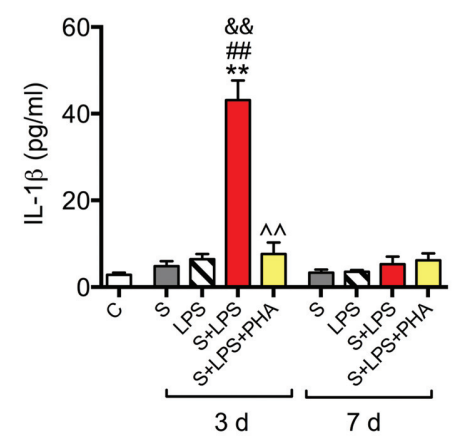

B



Figure 4. Infection prolongs systemic and neuroinflammatory response after surgery. Mice were injected with LPS $24 \mathrm{~h}$ after orthopedic surgery. Plasma levels of IL-1 $\beta$ and IL-6 were measured by ELISA in plasma and hippocampal tissue. (A) At 3 d following surgery, LPS treated animals had a sustained elevation of IL-1 $\beta$ in plasma. (B) Similarly, systemic levels of IL-6 were also upregulated at $3 \mathrm{~d}$, returning to baseline by $7 \mathrm{~d}$. Data are expressed as mean \pm SEM and compared by one-way ANOVA and Student-Newman-Keuls method, $\mathrm{n}=4\left({ }^{* \star} P<0.01\right.$ versus $\mathrm{C}$; ${ }^{\#} P<0.05$ versus $\mathrm{S}$; ${ }^{\# \#} P<0.01$ versus $\mathrm{S}$; ${ }^{\& \&} P<0.01$ versus LPS; ${ }^{\wedge} P<$ 0.01 versus S+LPS). C, control; S, surgery.

LPS (100 ng/mL) to measure phosphorylation and nuclear translocation of the p65 subunit of NF-кB. Consistent with previous studies, $\alpha 7 \mathrm{nAChR}$ signaling prevented NF- $\mathrm{BB}$ activation in vitro (Figure 5A). Furthermore, cholinergic stimu- lation also prevented LPS-induced nicotinamide adenine dinucleotide phosphate (NADPH) oxidase production in vitro, restoring superoxide levels to baseline $(P<0.001$, Figure 5B). This suggests that $\alpha 7 \mathrm{nAChR}$ stimulation exerts antiinflam-
A



\section{B}

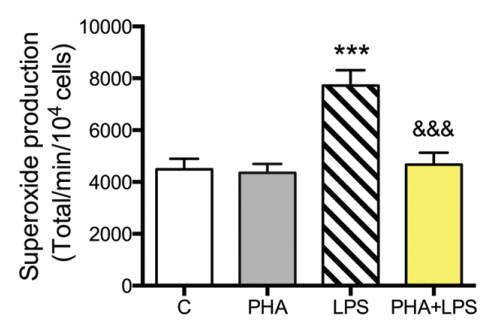

Figure 5. LPS-induced NF-кB and NADPH activation in BMDMs is blocked by PHA 568487. (A) Cultured BMDMs were stimulated with LPS (100 ng/mL) for $2 \mathrm{~h}$ and immunostained for nuclear phosphorylated NF-кB subunit p65. Preincubation with PHA $568487(10 \mu \mathrm{g} / \mathrm{mL})$ for 30 min significantly reduced activation and nuclear translocation of p65 (LPS+PHA). NF- $\mathrm{kB}$ activation was undetectable in unexposed cells (medium) or in cells exposed to PHA 568487 (PHA). (B) NADPH oxidase mediated superoxide generation from BMDMs was measured by chemiluminescence method. LPS stimulation at $10 \mathrm{ng} / \mathrm{mL}$ for $24 \mathrm{~h}$ significantly increased superoxide generation from BMDMs ( ${ }^{* *} P<0.001$ versus $C$ ), which was abolished by preincubation of PHA ( ${ }^{\& \&} P<0.001$ versus LPS). PHA per se did not significantly affect superoxide generation in nonstimulated BMDMs. Data are expressed as mean \pm SEM and compared by one-way ANOVA and Bonferroni post hoc, $n=6$. C, control; PHA, PHA 568487

matory and antioxidative effects on macrophages, thus preventing further proinflammatory cytokines release.

We next assessed if PHA 568487 would affect the rate of bone healing. Bone repair occurs through a process resembling endochondral bone formation, which begins with a cartilaginous intermediate (23). We differentiated a chondrogenic cell line in the presence of PHA 568487 or vehicle and found that PHA 568487 reduced the rate of deposition of cartilaginous extracellular matrix (based on Alcian blue staining of glycosaminoglycans) (Figure 6A). After $7 \mathrm{~d}$ in the presence of PHA 568487 there was a reduction in the number of Alcian blue-positive colonies ( $62 \%$ of vehicle, $p=0.0119, \mathrm{n}=6$ wells). This was confirmed by assessing total glycosaminoglycan labeling, which was greatest after $12 \mathrm{~d}$ of differentiation when it reached a $64 \%$ reduction versus the vehicle (Figure 6B).

\section{DISCUSSION}

In this study we demonstrated that postoperative complications such as infection prolong the neuroinflammatory response after surgical trauma and worsen cognitive decline. Stimulation of the cholinergic antiinflammatory pathway reduces the innate immune response offering a novel strategy to prevent cognitive decline following postoperative endotoxemia.

Nonresolving inflammation is recognized as a major component of several disease states (24). Aseptic injury activates the innate immune system by releasing damage-associated molecular patterns (DAMPs) and cytokines, which interfere with the brain microenvironment and may ultimately lead to cognitive decline. In this study, we combined the effects of aseptic injury with postoperative endotoxemia using LPS as a key component of the gram-negative bacteria outer membrane and a classic initiator of the inflammatory cascade. Although LPS does not faithfully reproduce the complexity of in-hospital infections, especially due to the higher incidence of polymicrobial and virulent strains of 
A

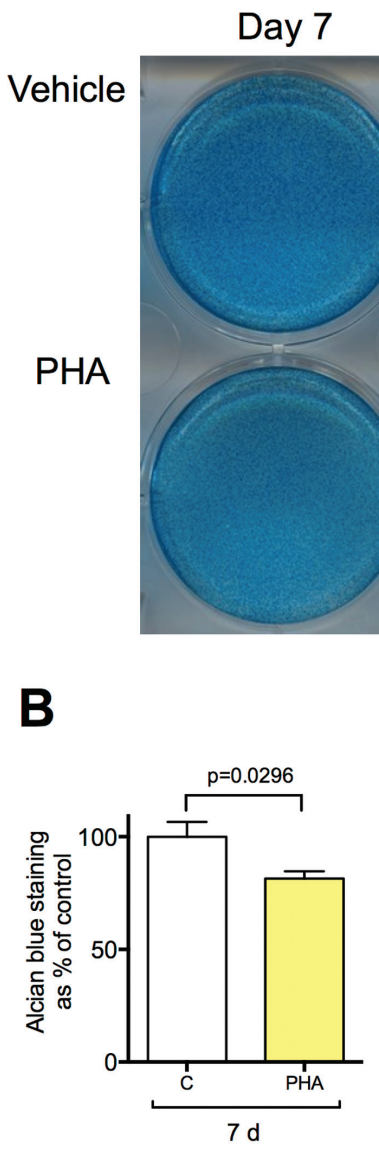

Day 12
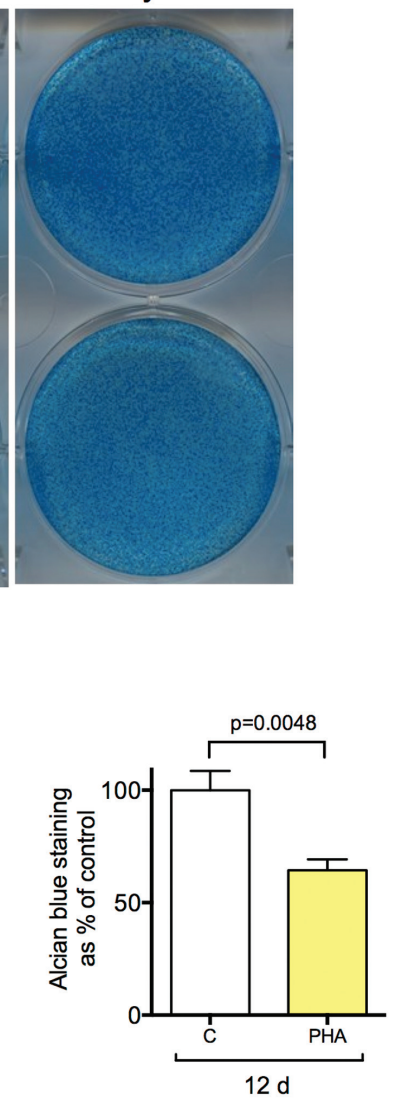

Figure 6. PHA 568487 impairs cartilage matrix formation. (A) C5.18 cells were differentiated in the presence or absence of $10 \mu \mathrm{g} / \mathrm{mL}$ PHA, and fixed at two time points. (B) Alcian blue staining was performed on whole wells, which were leached and quantified. Data are expressed as mean \pm SEM and compared by t test, $n=6$. C, control; PHA, PHA 568487.

methicillin-resistant S. aureus (MRSA) (25), gram-negative bacteria commonly associate with orthopedic blast injuries (26). There is also opportunity for LPS to bind to orthopedic implants and biomaterials, potentially triggering a systemic inflammatory reaction and prompting joint revision surgery (27), thus these results may be applicable to different perioperative settings.

Augmented levels of systemic cytokines can account for many of the physiological and behavioral changes, both in humans and animals, and are dysregulated in multiple disease states. The effects of additive systemic inflammatory insults in this mouse model are evident, both in the neuroinflammatory response and hippocampal-dependent memory function assessed by contextual fear conditioning. Systemic inflammation and release of proinflammatory cytokines including IL-1 $\beta$ and IL- 6 after surgery can prime the CNS, causing microglia to become more susceptible to the second hit (28). Both microglia priming and synaptic loss have been related to acute memory dysfunction and neurodegenerative processes in the presence of a deleterious systemic proinflammatory milieu (29). Similar changes in CNS function, including synaptic plasticity and altered neurogenesis, have been observed during aging, with specific age-related systemic chemokines contributing to the overall processes of cognitive decline
(20). Even though cognitive impairments are observed in patients at all ages, only the elderly are at higher risk for POCD (30) and the priming of the immune system in the aged may be responsible for this higher susceptibility and more severe prognosis.

The mechanisms whereby cytokines and other proinflammatory mediators prime the CNS after surgery are not fully understood. LPS does not cross the BBB (31) but has the ability to disrupt immune-endothelial interactions, thus allowing proinflammatory immune cells to enter the CNS (32). Recently, we reported a key role for macrophage infiltration in the hippocampus after orthopedic surgery through a disrupted BBB $(12,21)$, suggesting both cytokines and LPS may access the brain directly following the initial trauma. Remarkably, similar effects on cognitive decline were reported using a subclinical dose of LPS administered systemically before surgery, demonstrating that the reversed combination of these two hits also impaired hippocampal memory consolidation (33).

Proinflammatory cytokines also can stimulate afferent C-fibers to open the blood-spinal cord barrier allowing macrophages, T-cells and other soluble factors to reach the brain via the spinal cord $(34,35)$. Interestingly, patients undergoing total hip arthroplasty display elevated proinflammatory cytokines in the cerebrospinal fluid (CSF) in the postoperative period and this could contribute to worse cognitive outcome (36). Biomarkers of acute neuroinflammation and Alzheimer's disease (AD) including IL-6, TNF- $\alpha$, S100 $\beta$ and tau are similarly regulated in the CSF of otherwise healthy patients following surgery under general anesthesia (37). Serum levels of IL-6 also have been correlated with the diagnosis of periprosthetic infection after arthroplasty (38). In our animal model, the systemic levels of IL-6 also were significantly elevated after surgery and LPS challenge, indicating a possible role for biomarkers in predicting poorer prognosis including adverse cognitive outcome. Cholinergic stimulation limits systemic 


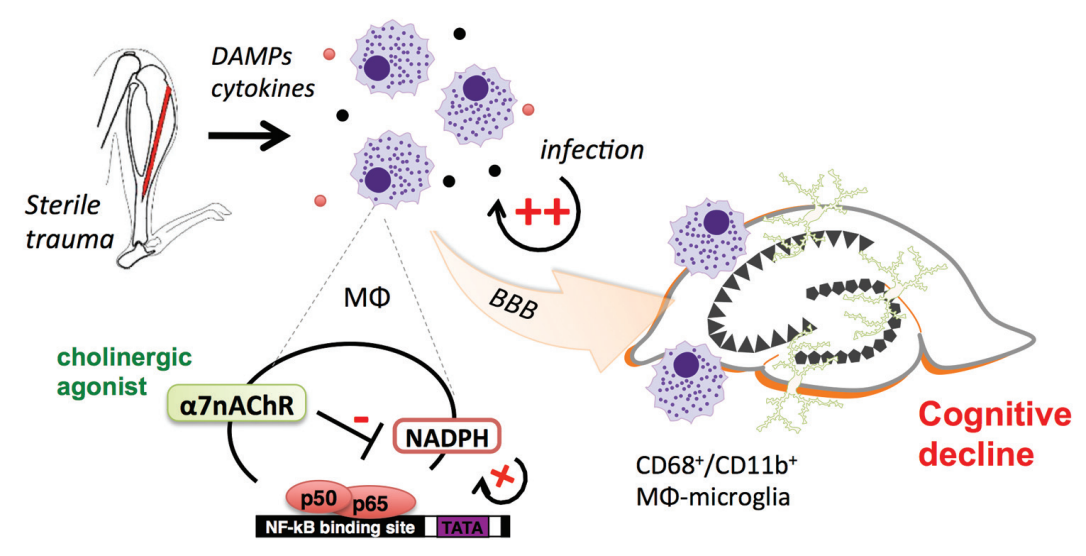

Figure 7. Working model. Peripheral injury leads to a systemic inflammatory response and release of danger-associated molecular patterns (DAMPs) in the circulation causing neuroinflammation and subsequent cognitive decline. Postoperative infection elicits a secondary hit that exacerbates the proinflammatory milieu and CNS dysfunction. Stimulation of the cholinergic antiinflammatory signaling pathway via selective $\alpha 7 \mathrm{nAChR}$ improves cognitive decline though modulation of NF- $\mathrm{kB}$ and oxidative stress activity on monocytes, thus attenuating neuroinflammation and dampening the systemic proinflammatory milieu.

proinflammatory cytokine release which may stabilize endothelial function at the $\mathrm{BBB}$ and thereby protect the brain from prolonged neuroinflammation and cognitive dysfunction $(12,39)$.

Apart from the cellular and humoral mediation in immune-to-brain signaling, neural regulation of inflammatory processes occurs in many disorders (40). In this study, we tested the effects of a selective $\alpha 7 \mathrm{nAChR}$ agonist on the nonresolving inflammation following surgery and LPS. Systemic administration of PHA 568487 after LPS exposure reduced proinflammatory cytokines and neuroinflammation, significantly attenuating

$\mathrm{CD} 11 \mathrm{~b}^{+} / \mathrm{CD}^{+} 8^{+}$cells in the hippocampus and improving cognitive function. It is possible that the antiinflammatory effects of PHA 568487 are not limited to NF-кB and oxidative stress signaling in bone marrow-derived monocytes as we report in this study (Figure 7). $\alpha 7 \mathrm{nAChR}$ signaling was recently shown to modulate inflammasome activity, affecting the proteolytic maturation of key cytokines such as IL-1 $\beta$, IL-18 and HMGB-1 (41). Notably, both IL-1 $\beta$ and HMGB-1 have been related to cognitive decline in models of surgery $(9,10,42)$, infection (43) and sepsis (44), suggesting targeting these molecules may protect against cognitive decline.
Cholinergic agonists, acting on both $\alpha 7$ and $\alpha 4 \beta 2$ subunits, can also alter synaptic plasticity, enhancing neuronal transmission and improving cognitive function (45). Notably, $\alpha 7 \mathrm{nAChR}$ also is functionally expressed on nonexcitable cells, including the endothelium, keratinocytes and chondrocytes (46). Using a chondrogenic cell line model, we found PHA 568487 impaired cartilage matrix formation, which may indicate a disruption of the bone healing process after an orthopedic trauma. Prolonged nicotine exposure was earlier shown to delay skeletal growth through $\alpha 7 \mathrm{nAChR}$-dependent signaling (47). These findings warrant further studies in vivo to ascertain the potential benefits of cholinergic modulation in the perioperative setting.

Recently, a role for the vagus nerve in regulating proresolving mediators, such as resolvin D1, has been proposed and this provides a novel understanding for the neuronal control of resolution (48). Interestingly, administration of aspirintriggered resolvin D1 after surgery protected the brain from synaptic dysfunction and cognitive decline, suggesting that stimulation of endogenous signaling pathways may represent an effective way to prevent and treat inflammatory conditions by promoting resolution $(14,49)$. Of relevance to POCD, aberrant inflammation and impaired proresolving mediator levels have been associated with the process of physiological aging (50), and may provide novel approaches to biomarkers discovery in aging patients at risk for cognitive decline.

\section{CONCLUSION}

In conclusion, this study provides novel insights into the impact of aseptic trauma and LPS on brain function. Through stimulation of the cholinergic antiinflammatory pathway, we may offer a novel strategy to limit POCD and prevent adverse neuroinflammatory complications. Excessive inflammation and oxidative stress represent novel targets for resolving neuroinflammation in the perioperative period.

\section{ACKNOWLEDGMENTS}

This work was supported by R01 GM104194 (M Maze), the Anesthesia Department, UCSF (M Maze), a research grant program of the European Society of Anaesthesiology and Karolinska Institutet Funds (N Terrando), NIH grants R01 NS052189 and R21 NS082976

(K Akassoglou) and the Mathilda and Terence Kennedy Institute of Rheumatology Trust (M Feldmann, C Monaco).

\section{DISCLOSURE}

The authors declare that they have no competing interests as defined by Molecular Medicine, or other interests that might be perceived to influence the results and discussion reported in this paper.

\section{REFERENCES}

1. Ehlenbach WJ, et al. (2010) Association between acute care and critical illness hospitalization and cognitive function in older adults. JAMA. 303:763-70.

2. Saczynski JS, et al. (2012) Cognitive trajectories after postoperative delirium. N. Engl. J. Med. 367:30-9.

3. Moller JT, et al. (1998) Long-term postoperative cognitive dysfunction in the elderly ISPOCD1 study. ISPOCD investigators. International Study of Post-Operative Cognitive Dysfunction. Lancet. 351:857-61.

4. Terrando N, et al. (2011) Perioperative cognitive 
decline in the aging population. Mayo Clin. Proc. 86:885-93.

5. Riedel B, Browne K, and Silbert B. (2014) Cerebral protection: inflammation, endothelial dysfunction, and postoperative cognitive dysfunction. Curr. Opin. Anaesthesiol. 27:89-97.

6. Tracey KJ. (2007) Physiology and immunology of the cholinergic antiinflammatory pathway. J. Clin. Invest. 117:289-96.

7. Dantzer R, et al. (2008) From inflammation to sickness and depression: when the immune system subjugates the brain. Nat. Rev. Neurosci. 9:46-56.

8. Terrando N, et al. (2010) Tumor necrosis factoralpha triggers a cytokine cascade yielding postoperative cognitive decline. Proc. Natl. Acad. Sci. U. S. A. 107:20518-22.

9. Cibelli M, et al. (2010) Role of interleukin-1beta in postoperative cognitive dysfunction. Ann. Neurol. 68:360-8.

10. Vacas S, et al. (2014) High-mobility group box 1 protein initiates postoperative cognitive decline by engaging bone marrow-derived macrophages. Anesthesiology. 120:1160-7.

11. Olofsson PS, et al. (2012) Rethinking inflammation: neural circuits in the regulation of immunity. Immunol. Rev. 248:188-204.

12. Terrando N, et al. (2011) Resolving postoperative neuroinflammation and cognitive decline. Ann. Neurol. 70:986-95.

13. Harry LE, et al. (2008) Comparison of the healing of open tibial fractures covered with either muscle or fasciocutaneous tissue in a murine model. J. Orthop. Res. 26:1238-44.

14. Terrando N, et al. (2013) Aspirin-triggered resolvin D1 prevents surgery-induced cognitive decline. FASEB J. 27:3564-71.

15. Spagnoli A, et al. (2001) Antiproliferative effects of insulin-like growth factor-binding protein-3 in mesenchymal chondrogenic cell line RCJ3.1C5.18. relationship to differentiation stage. J. Biol. Chem. 276:5533-40.

16. Lunstrum GP, et al. (1999) Chondrocyte differentiation in a rat mesenchymal cell line. J. Histochem. Cytochem. 47:1-6.

17. Newton PT, et al. (2012) Chondrogenic ATDC5 cells: an optimised model for rapid and physiological matrix mineralisation. Int. J. Mol. Med. 30:1187-93.

18. Perego C, Fumagalli S, and De Simoni MG. (2011) Temporal pattern of expression and colocalization of microglia/macrophage phenotype markers following brain ischemic injury in mice. J. Neuroinflammation. 8:174.

19. Wang $H$, et al. (2003) Nicotinic acetylcholine receptor alpha7 subunit is an essential regulator of inflammation. Nature. 2003. 421:384-8.

20. Villeda SA, et al. (2011) The ageing systemic milieu negatively regulates neurogenesis and cognitive function. Nature. 477:90-4.

21. Degos V, et al. (2013) Depletion of bone marrowderived macrophages perturbs the innate immune response to surgery and reduces postoper- ative memory dysfunction. Anesthesiology. 118:527-36.

22. Olofsson PS, et al. (2012) $\alpha 7$ nicotinic acetylcholine receptor $(\alpha 7 \mathrm{nAChR})$ expression in bone marrow-derived non- $T$ cells is required for the inflammatory reflex. Mol. Med. 18:539-43.

23. Ford JL, Robinson DE, Scammell BE. (2004) Endochondral ossification in fracture callus during long bone repair: the localisation of 'cavity-lining cells' within the cartilage. J. Orthop. Res. 22:368-75.

24. Nathan C, Ding A. (2010) Nonresolving inflammation. Cell. 140:871-82.

25. Bernthal NM, et al. (2010) A mouse model of post-arthroplasty Staphylococcus aureus joint infection to evaluate in vivo the efficacy of antimicrobial implant coatings. PLoS One. 5:e12580.

26. Mody RM, et al. (2009) Infectious complications of damage control orthopedics in war trauma. J. Trauma. 67:758-61.

27. Ragab AA, et al. (1999) Measurement and removal of adherent endotoxin from titanium particles and implant surfaces. J. Orthop. Res. 17:803-9.

28. Perry VH, Holmes C. (2014) Microglial priming in neurodegenerative disease. Nat. Rev. Neurol. 10:217-24.

29. Cunningham C, et al. (2009) Systemic inflammation induces acute behavioral and cognitive changes and accelerates neurodegenerative disease. Biol. Psychiatry. 65:304-12.

30. Monk TG, et al. (2008) Predictors of cognitive dysfunction after major noncardiac surgery. Anesthesiology. 108:18-30.

31. Gyoneva S, et al. (2014) Systemic inflammation regulates microglial responses to tissue damage in vivo. Glia. 62:1345-60.

32. Ramirez SH, et al. (2012) Activation of cannabinoid receptor 2 attenuates leukocyte-endothelial cell interactions and blood-brain barrier dysfunction under inflammatory conditions. J. Neurosci. 32:4004-16

33. Fidalgo AR, et al. (2011) Systemic inflammation enhances surgery-induced cognitive dysfunction in mice. Neurosci. Lett. 498:63-6.

34. Beggs S, et al. (2010) Peripheral nerve injury and TRPV1-expressing primary afferent $\mathrm{C}$-fibers cause opening of the blood-brain barrier. Mol. Pain. 6:74.

35. Arima Y, et al. (2012) Regional neural activation defines a gateway for autoreactive $\mathrm{T}$ cells to cross the blood-brain barrier. Cell. 148:447-57.

36. Buvanendran A, et al. (2006) Upregulation of prostaglandin E2 and interleukins in the central nervous system and peripheral tissue during and after surgery in humans. Anesthesiology. 104:403-10.

37. Tang JX, et al. (2011) Human Alzheimer and inflammation biomarkers after anesthesia and surgery. Anesthesiology. 115:727-32.

38. Di Cesare PE, et al. (2005) Serum interleukin-6 as a marker of periprosthetic infection following total hip and knee arthroplasty. J. Bone Joint Surg. Am. 87:1921-7.
39. Krafft PR, et al. (2013) PHA-543613 preserves blood-brain barrier integrity after intracerebral hemorrhage in mice. Stroke. 44:1743-7.

40. Andersson U, Tracey KJ. (2012) Neural reflexes in inflammation and immunity. J. Exp. Med. 209:1057-68.

41. Lu B, et al. (2014) Alpha 7 nicotinic acetylcholine receptor signaling inhibits inflammasome activation by preventing mitochondrial DNA release. Mol. Med. 20:350-8

42. He HJ, et al. (2012) Surgery upregulates high mobility group box-1 and disrupts the blood-brain barrier causing cognitive dysfunction in aged rats. CNS Neurosci. Ther. 18:994-1002.

43. Terrando N, et al. (2010) The impact of IL-1 modulation on the development of lipopolysaccharide-induced cognitive dysfunction. Crit. Care. 14:R88.

44. Chavan SS, et al. (2012) HMGB1 mediates cognitive impairment in sepsis survivors. Mol. Med. 18:930-7.

45. Couey JJ, et al. (2007) Distributed network actions by nicotine increase the threshold for spike-timing-dependent plasticity in prefrontal cortex. Neuron. 54:73-87.

46. Sharma G, Vijayaraghavan S. (2002) Nicotinic receptor signaling in nonexcitable cells. J. Neurobiol. 53:524-34.

47. Kawakita A, et al. (2008) Nicotine acts on growth plate chondrocytes to delay skeletal growth through the alpha7 neuronal nicotinic acetylcholine receptor. PLoS One. 3:e3945.

48. Mirakaj V, et al. (2014) Vagus nerve controls resolution and pro-resolving mediators of inflammation. J. Exp. Med. 211:1037-48.

49. Serhan CN, et al. (2007) Resolution of inflammation: state of the art, definitions and terms. FASEB J. 21:325-32.

50. Arnardottir HH, et al. (2014) Aging delays resolution of acute inflammation in mice: reprogramming the host response with novel nanoproresolving medicines. J. Immunol. 193:4235-44. 